\title{
FACING THE CHALLENGES FROM DIFFERENT REALITIES: E-LEARNING APPROACHES FOR AFRICA AND EUROPE
}

\author{
Bruno de Sousa and Dulce Gomes \\ University of Coimbra, Faculty of Psychology and Education Sciences, CINEICC \\ University of Évora, Department of Mathematics, School of Science and Technology, CIMA/UE, \\ bruno.desousa@fpce.uc.pt
}

Given that the UN's Millennium Development Goals focus on primary education, Guy Pfefferman has observed how higher education was omitted within the African context. He also found that skills development for employment requires a radical change, advocating for eLearning as the only way to accomplish this in a meaningful and relevant way. In Africa we have the fastest growing population in the world with $40 \%$ under the age of 15 yet with one of the lowest higher education enrollments in the world, whereas in Europe we note an ageing corps of teachers, who despite being identified as having more experience, tend to suffer from technophobia. How to face the challenge of profiting from this experience and improve teaching practices when no more than $14 \%$ of the African population has access to internet? Simple ideas from a pilot study will be presented in order to overcome problems that may arise in less than perfect environments.

\section{WHY BOTHER WITH STATISTICS?}

Statistics play a crucial role in transforming information into educated knowledge and thus into efficient and effective decision-making. The informed knowledge of a country's statistics enhances the promotion and participation of young people and adults in building more inclusive and social conscious countries. As early as the 19th century Thomas Carlyle recognized the role of Statistics in the defense of citizenship by saying "[...] a judicious man looks at statistics [...] to save himself from having ignorance foisted on him." (Chartism, 1839).

With the proliferation of information that inundates us through the media or the enormous expansion of social networks, it is becoming increasingly urgent for citizens to have a strong statistical literacy associated with equally strong media literacy. As Francisco, K. (2010) mentioned "The old receivers are now playing the role of the emitters, with their voices echoing through the social networks of which they are part."

Statistics can then be seen as a "[...] shield of Citizenship. It is an essential element in the social contract, in overcoming mistrust between States [...] in how citizens, with an informed public opinion, limit the abuse of State powers, or how, for example, it can work as a defense of the powers of the State against the (perversion of) public opinion by the media. Of course, like everything else, it can have a perverse and abusive use, contributing instead to giving an appearance of solidity and objectivity to lies [...]" (Pestana, D., 2007).

According to PARIS21 STRATEGY: 2016-2020 (Partnership in Statistics for Development in the 21th century), organized around the motto "Improving lives through better statistics", "[...] there was a critical and urgent need to help developing countries produce more and better data to underpin the Millennium Development Goals (MDGs), build a culture of evidence-based decision-making, and strengthen accountability and good governance. [...] Making progress towards the Sustainable Development Goals will require not only better coordination of policy agendas, but also of statistical programs to support monitoring and decision making at all levels - national, regional, and global" (http://www.paris21.org/Strategy_2016-2020).

Therefore, all citizens must have at least basic knowledge of statistics that enables them to carry out their professional, social and personal activities. And that qualifies them to know how to interpret, reflect, criticize and communicate information and statistical concepts.

\section{THE REALITY}

Given that the UN's Millennium Development Goals focused on primary education, Guy Pfefferman (Manji et al., 2015) observed how higher education was omitted. He also found that skills development for employment requires a radical change, advocating for eLearning as the only way to accomplish this in a meaningful and relevant way. How to improve teaching practices when less than $14 \%$ of the African population has access to internet (Manji et al., 2015)? With a median 
population age of 20 and enrollment in higher education one of the lowest in the world, Africa needs to apply ICT (Information and Communications Technology) creatively to improve both access to and quality of education (AAI, 2015).

Europe, with an ageing corps of teachers and a radical decrease in the number of children, faces different challenges (Schlotter et al., 2008). Although older teachers may be identified as having more experience, they are also tend to suffer from technophobia. Additionally, students in long, traditional face-to-face classes are increasingly distracted by social-networks. A change in teaching methodology is needed, especially given analysts' prediction that by 2020 higher education will be responsible for a market share in eLearning of more than $64 \%$ in Europe (TechNavio, 2016).

For historical reasons, Portugal has been a country of choice for many students within the Community of Portuguese Language Countries (CPLP). In our more than ten years of experience in teaching CPLP students, we have seen that, in addition to the problems inherent to any adaptation to and integration into a foreign country, there are still some barriers to overcome. According to data from UNICEF (Monitoring the Situation of Children and Women, 2016), Brazil ranks second in the CPLP area, after Portugal, in terms of literacy rate $(90 \%$ of adult Brazilians can read and write: $90 \%$ of men and $91 \%$ of women). Mozambique has the lowest rate: only half of Mozambicans can read and write, at 51\% (of whom $67 \%$ are men and $36 \%$ women). In both Guinea-Bissau and East-Timor, these rates are 58\% (70\% men and $45 \%$ women; and 64\% men and $53 \%$ women, respectively). In Angola and São Tome and Principe, $70 \%$ of adults can read and write $(80 \%$ men and $60 \%$ women) while in Cape Verde, this rate is $85 \%(90 \%$ men and $80 \%$ women). In addition to these differences, the educational systems and opportunities among CPLP countries differ; thus, technical and scientific barriers need to be addressed in order to guarantee the inclusion and well-being of these young people when confronted with the Portuguese/European educational system. In the past, attempts have been made by what has been called a PreparationYear (Ano Zero), but with limited success due to diversity still existing within these students. We believe that a more custom-made approach, one respecting a student's own rhythm, will contribute to a greater integration of students into the host institution, to their academic success, and will naturally generate more lasting bonds between countries.

In light of this, our approach will be based on communication and information technologies. A pilot-project, in São Tomé and Príncipe and Portugal (Coimbra), will address how to overcome the distance between students and teachers resulting from systematic connectivity problems in a b-learning environment, and how working in a computer language, $\mathrm{R}$ ( $\mathrm{R}$ Core Team, 2016), students can overcome some of the language barriers that may exist between these countries. In particular, we will explore how social-networks such as Facebook and videotaping can be helpful in addressing these issues within a student-centered learning environment.

\section{OVERCOMING PROBLEMS IN LESS THAN PERFECT ENVIRONMENTS}

Avoiding students' isolation that necessarily leads to drop outs marks the success of any educational interventions when using e- or b-learning. Teacher-Student bonds grow stronger when there is some regularity in the synchronous sessions, although they require nearly constant attention on the part of the teacher.

Adding to this fact are the limitations in assessing communication technologies in some African countries such as Angola and São Tomé and Principe (just to mention two of the countries with which we have worked), or simply other realities such as the lack of electricity on a daily basis, these obstacles making it very difficult to maintain an active, ongoing and successful distance-learning environment, whether talking on Skype or using a more sophisticated video conferencing approach.

We will start by presenting a way to avoid the stress generated from inconsistent communication systems, followed by a simple way to maintain students' engagement in longdistance learning experiences.

\section{Facebook, one way to do it}

Although the use of the Internet is still limited in some CPLP countries, ranging from $4.3 \%$ in Guinea-Bissau to $67.7 \%$ in Portugal, Facebook use is over $75 \%$ in all countries, with the 
exception of Angola at 58.8\% (http://www.internetworldstats.com/), thus making Facebook a natural choice as an ally in overcoming some of the problems in e-Learning experiences.

By creating a Facebook page and a group, students have access to course-related materials such as videos, articles, assignments, or any PDF, Word or Excel documents (Figure 1).
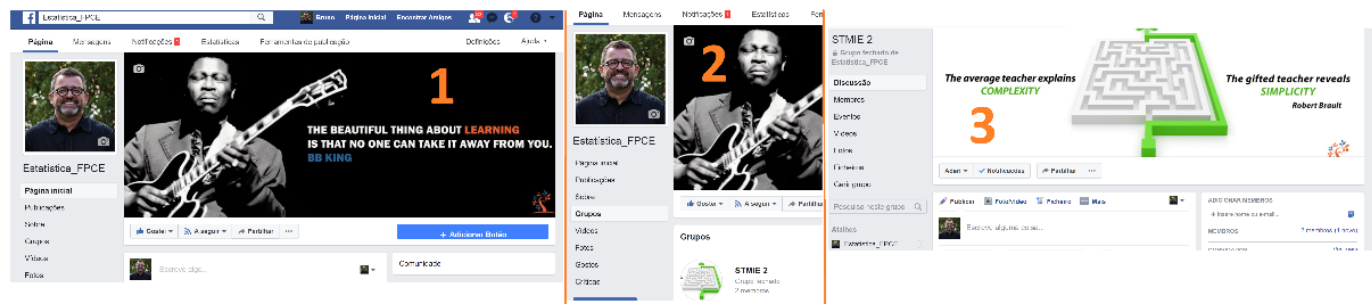

Figure 1: A Facebook page for a Statistical Course in 3 steps.

Facebook will emulate any course website, with the advantage of creating a sense of virtual community, one that students are connected to and can participate in via ongoing online discussions.

The difficulties that often occur in synchronized online sessions where constant interruptions occur will be minimized through virtual chatting. This approach will create the sense of a class discussion where students and teacher are physically present, without the need for a constant connectivity to the Internet. In addition, teachers and students can use some of the tools available in Facebook to enhance this communication. For example, a teacher can instantly tape his/her answer to some recurrent question made by students and upload it in real time (Figure 2).

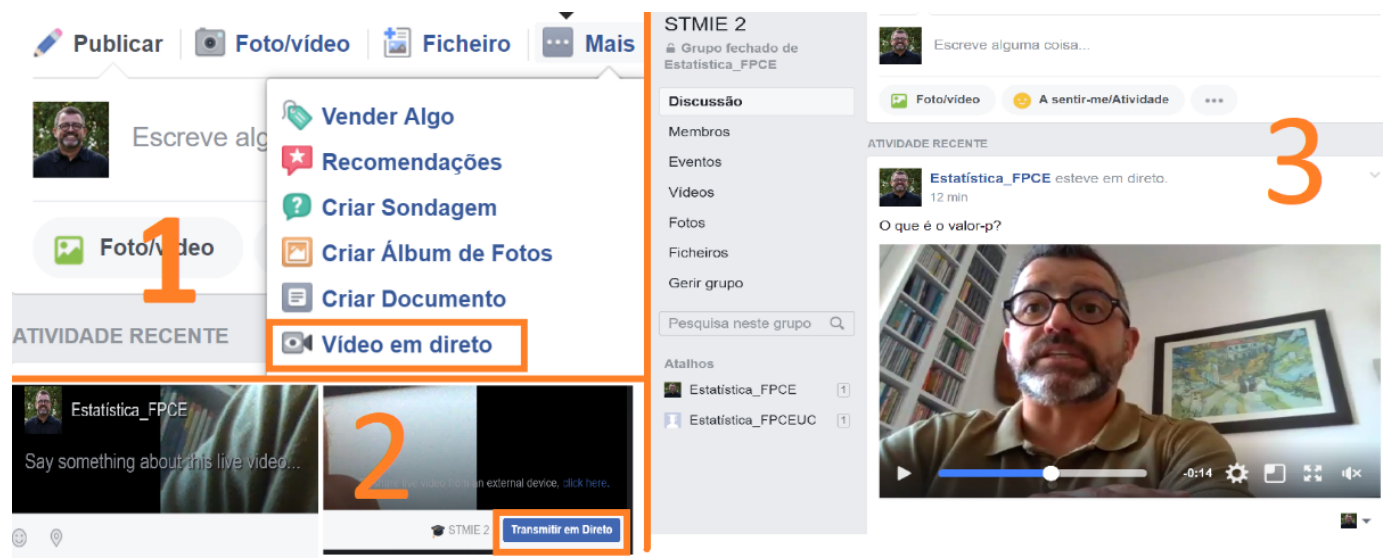

Figure 2: Taping an answer to students' questions and uploading it in Facebook in 3 steps.

There are endless opportunities with TED-Ed (http://ed.ted.com/) in creating challenges, for example when analyzing data with SPSS, in order to flip a classroom.

With so many people being familiar with Facebook, we believe this approach will optimize the number of teacher orientation sessions and avoid the frustration of long ineffective synchronized online sessions that, in many cases, lead to drop-outs and the isolation of students.

These examples show how very simple ideas allied with the use of technology can facilitate the implementation of e-Learning in countries with less than perfect conditions. 


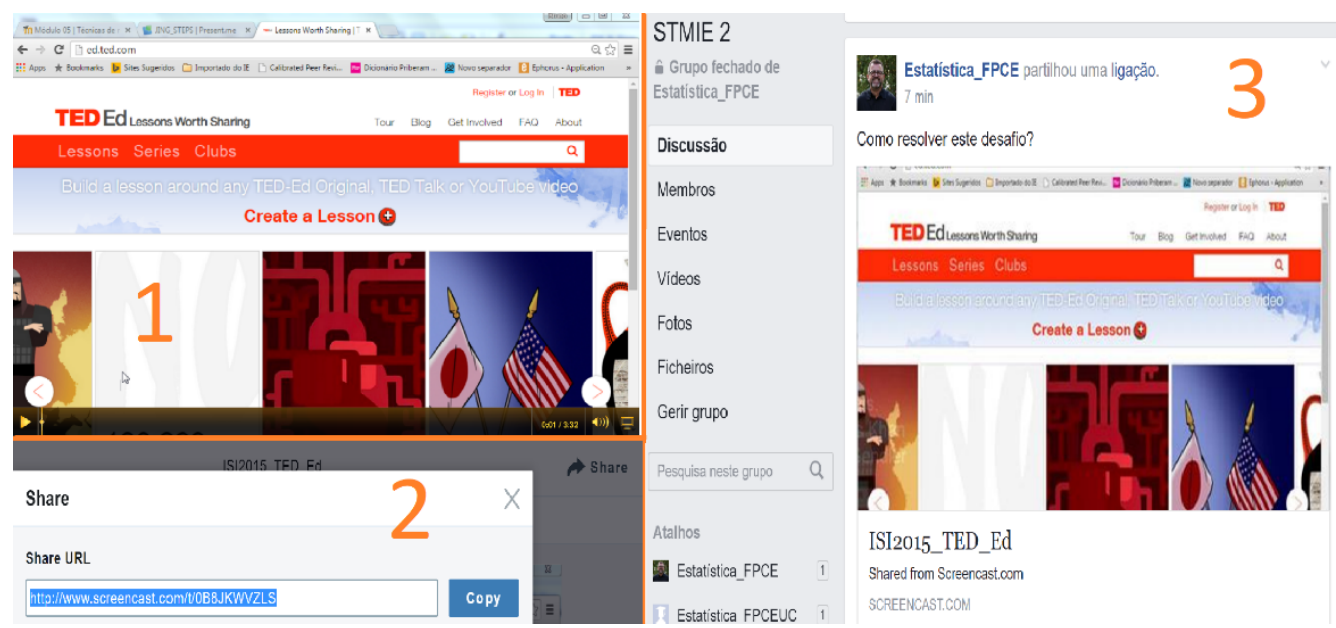

Figure 3: Uploading a TED-Ed lesson in Facebook in 3 steps.

\section{The Facilitator}

There is a real need for a teacher to be able to ensure his/her presence in distance-learning, a role harder to achieve with a large number of students. One way to successfully accomplish these goals is to get students involved in this process as facilitators. Thus, one can create a Facebook Group, where one student will be nominated as the "Team Leader", where all together they will need to solve, for example, the following task:

Task: Consider a variable of interest $\mathrm{X}$ following a normal distribution with mean 2 and variance 4 . We would like to investigate some properties of the sample mean and variance. For that, perform $\mathrm{n}$ simulations of the variable $\mathrm{X}$ in $\mathrm{R}$ command and calculate the sample mean and variance.

- Choose different values for $\mathrm{n}$, and verify if the sample mean and variance are unbiased estimators for the population mean and variance, respectively.

- Verify what happens with the variance of the sample mean when the number of simulations increases.

- Perform 10 and 100 simulations of the variable X and study the distribution of the sample mean. Obtain a histogram and, in each case, perform a test of normality. What do you expect to see?

During the process of completing this task, the Team Leader will be responsible for the interaction and possible face-to-face meetings within team members and the teacher, promoting discussions and problem solving sessions that will lead to the final solutions of the task. This not only will have the advantage of optimizing the number of teacher orientation sessions and avoiding the frustration of long ineffective synchronized online sessions, but also would promote the cooperatively built relationships between team members, so necessary for a success in an elearning environment. The interactions with the teacher could be done through the use of many of the functions available in Facebook such as taping an instant video with the replies to some of the questions that will arise among the group, or make available any documents or videos on YouTube that will might help to successfully complete the task.

By performing this challenge through $\mathrm{R}$, students will guarantee that their work will be accessible to all users in the future. The $\mathrm{R}$ language is a powerful tool to program statistical techniques in a straightforward and simple way. Apart from being totally free, $\mathrm{R}$ is constantly updated through the contribution of several researchers on the most current statistical methods, and it is a language compatible with several platforms such as Windows, Unix, MacOs. In addition to these more technical advantages, even in the CPLP communities, students do have different language skills, thus working in a computer language such as $\mathrm{R}$, helps students to overcome this barrier, lessening their isolation.

At the end of each challenge, the achievements of the team leader could be rewarded in terms of his/her evaluation, and this leadership role could rotate among the students. 


\section{FINAL COMMENTS}

Some of the main challenges to be faced when introducing technologies in learning experiences are:

- The initial work that is necessary for implementing the activities;

- How to change the learning culture of students and people in general;

- How to deal with different learning rhythms in a classrooms;

- What to do when students do not watch the videos.

Not only do students like multimedia contexts, their use also allows us to create a more personal environment, making it easier to pass along more information. In addition, once the first step is taken, anyone can access the materials on-line, generating greater interest from a much larger number of individuals. When used in a flipped classroom, it can reduce lecture time considerably and increase the tutorial time spent with students. At the end of the day, students enjoy the videos immensely and use them to review many of the statistical techniques being studied.

The strategies adopted in this pilot study contribute to the promotion of statistical literacy among students, as many of them are teachers in Mathematics on the secondary and university level. The alliance between statistical and digital literacy through the promotion of new teachinglearning processes contributes to the improvement of adopted teaching methodologies, encouraging the implementation of the use of free software, namely $\mathrm{R}$, in the pedagogical practices of teachers and in daily life. Resulting from this is the promotion of greater student participation in a learning process that is more focused not only on their autonomous work, but also on the development of their abilities to work as a team, allowing for people from different cultures and with varying levels of Portuguese language skills to work and communicate with each other through a common mathematical language.

As Danai Nhando indicates (2015, retrieved from https:/elearningindustry.com/3-keychallenges-implementing-elearning-in-africa) there are 3 key challenges of implementing eLearning in Africa: Internet access and connectivity, availability of locally developed content and curriculum online, and training and professional development. The investment necessary for such implementations makes e-Learning in Africa a slow process. Simple ideas such as the ones presented here may contribute to addressing these challenges that, although requiring generations to be successfully solved, call for new guidelines in less than perfect environments. Future projects are in preparation in order to address the development of online contents for teacher training.

\section{REFERENCES}

AAI (2015). State of Education in Africa Report 2015. New-York: The Africa-America Institute. TechNavio (2016). E-Learning Market in Europe 2016-2020. URL: http://www.technavio.com/report/europe-education-technology-e-learning-market.

Carlyle, T. (1839). Chartism, 1904:125

Francisco, K. (2010). O jornalismo e as redes sociais: participação, inovação ou repetição de modelos tradicionais? URL: http://revistas.ua.pt/index.php/prismacom/article/view/754

Manji, F., Jal, E., Badisang, B., \& Opoku-Mensah, A. (2016). The eLearning Africa Report 2015. Berlin: ICWE GmbH.

Pestana, D. (2007). Boletim de Primavera da Sociedade Portuguesa de Estatística. Sociedade Portuguesa de Estatística. Lisboa. URL: http://www.spestatistica.pt/images/boletim/boletimspe-primavera-07.pdf

R Core Team (2016). R: A language and environment for statistical computing. R Foundation sfor Statistical Computing, Vienna, Austria. URL: https://www.R-project.org/.

Schlotter, M., Schwerdt, G., \& Woessmann, L. (2008). The Future of European Education and Training Systems: Key Challenges and their Implications. EENEE Analytical Report No. 3 prepared for the European Commission.

UNICEF (2016). Data: Monitoring the Situation of Children and Women. URL: https://data.unicef.org/topic/education/overview/ 